\title{
Multiple Second Order Generalized Integrators for Harmonic Synchronization of Power Converters
}

\author{
Pedro Rodriguez \\ Member, IEEE \\ Technical University \\ of Catalonia \\ C/ Colom 1 08222, \\ Terrassa, Spain \\ prodriguez@ee.upc.edu
}

\author{
Alvaro Luna \\ Student Member, \\ IEEE \\ Technical University \\ of Catalonia \\ C/ Colom 1 08222, \\ Terrassa, Spain \\ luna@ee.upc.edu
}

\author{
Ion Etxeberría \\ Member, IEEE \\ IKERLAN \\ $\mathrm{P}^{\mathrm{o}}$ Arizmendiarrieta 2, \\ 20500 Mondragón, \\ Spain \\ ietxeberria@ikerlan.es
}

\author{
Juan R. Hermoso \\ Technical University \\ of Catalonia \\ C/ Colom 108222 , \\ Terrassa, Spain \\ hermoso@ee.upc.edu
}

\author{
Remus \\ Teodorescu \\ Senior Member, IEEE \\ Aalborg University \\ Pontoppidanstraede \\ 101, 9220 Aalborg, \\ Denmark \\ ret@iet.aau.dk
}

\begin{abstract}
This paper presents a new frequency-adaptive synchronization method for grid-connected power converters which allows estimating not only the positive- and negativesequence components of the power signal at the fundamental frequency, but also other sequence components at multiple frequencies. The proposed system is called the MSOGI-FLL since it is based on a decoupled network consisting of multiple second order generalized integrators (MSOGI) which are frequency-adaptive by using a frequency-locked loop (FLL). In this paper, the MSOGI-FLL is analyzed and its performance is evaluated by both simulations and experiments.
\end{abstract}

Index Terms-- Synchronization, Frequency-locked loops, Harmonic analysis, Frequency estimation, Monitoring.

\section{INTRODUCTION}

The electricity networks of the future will make an extensive use of power electronics devices and ICT applications [1], as an interface between the generator systems and the electrical network. One of the most important issues in the connection of power converters to the grid is the synchronization with the voltage at the point of common coupling (PCC). In three-phase systems, PLLs based on a synchronous reference frame (SRF-PLL) [2] have become a conventional synchronization technique. Although these systems have shown to be fast and accurate under balanced conditions its response is unacceptably deficient when the utility voltage is unbalanced.

Different advanced synchronization systems, able to deal with this grid conditions, have been proposed as an alternative to SRF-PLL such as the decoupled double synchronous reference frame PLL (DDSRF-PLL), the three phase enhanced PLL (3PH-EPLL) and the dual second order generalized integrator resting on a frequency-locked loop (DSOGI-FLL) was proven to be an effective solution under adverse grid conditions [3]-[9].

Although these advanced synchronization systems are capable to perform an accurate detection of the fundamental components of the voltage under unbalanced conditions they are unable to perform a more precise characterization of the power signals, something that is necessary in those applications where harmonics components, of either the voltage or the line current, should be controlled. Within this field a synchronization technique based on using adaptive notch filters for estimating multiple frequencies in a singlephase system was described in [9].

This paper proposes a new technique for detecting multiple frequency harmonics, with positive- and negative-sequences, in three-phase systems using an extended approach to that presented in [9]. The proposed system has been named as the MSOGI-FLL. In the following, the main building blocks of the MSOGI-FLL will be presented, putting a special attention on the description of the SOGI and the FLL and its applications for three phase systems. The discrete equation for implementing this system in digital signal processor will be presented as well. Finally the performance of the proposed system will be evaluated by both simulations and experiments.

\section{THE SECOND ORDER GENERALIZED INTEGRATOR}

An adaptive filter structure, based on the generalized integrator (GI) structure [10], named second order generalized integrator (SOGI) was formally presented in [11]-[12]. The adaptive filter based on the SOGI is shown in Fig. 1.

The two in-quadrature output signals of the filter of Fig. 1,

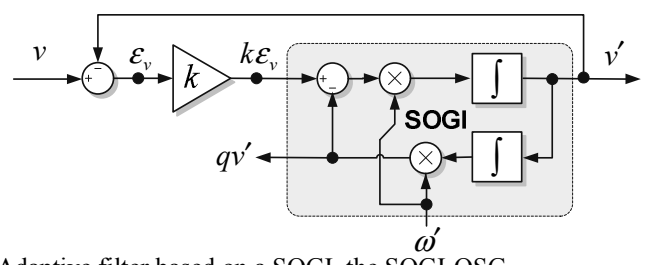

Fig. 1. Adaptive filter based on a SOGI, the SOGI-QSG.

$v$ ' and $q v$ ', are defined by the following transfer functions:

$$
\begin{aligned}
& D(s)=\frac{v^{\prime}}{v}(s)=\frac{k \omega^{\prime} s}{s^{2}+k \omega^{\prime} s+\omega^{\prime 2}} \\
& Q(s)=\frac{q v^{\prime}}{v}(s)=\frac{k \omega^{\prime 2}}{s^{2}+k \omega^{\prime} s+\omega^{\prime 2}}
\end{aligned}
$$


As it can be concluded from (1) the bandwidth of the bandpass filter is independent of the center frequency $\omega$ ', being exclusively set by the gain $k$. The same happens with the lowpass filter of (2), where the static gain only depends on the value of $k$. From (1) it can be concluded that the $q v$ ' output is always 90-degrees lagged from the $v$ ' output; independently of both the frequency of the $v$ input signal and the resonance frequency of the SOGI. For this reason, the adaptive filter of Fig. 1 was named as SOGI quadrature signal generator (SOGI-QSG).

\section{THE FREQUENCY LOCKED LOOP}

The frequency-locked loop (FLL) was presented in [13] as an effective mechanism for adapting the center frequency of the SOGI-QSG. The adaptive filter, including the FLL, is shown in Fig. 2.

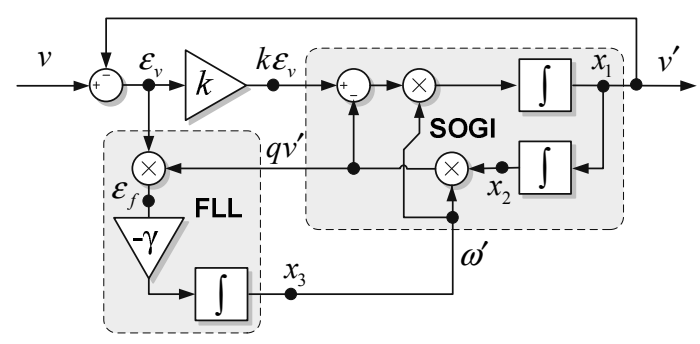

Fig. 2. The SOGI-FLL, a single-phase grid synchronization system.

The relationship between the in-quadrature output signal $q v$ ' and the error signal $\varepsilon_{v}$ should be analyzed in order to understand the behavior of the FLL. The transfer function from the input signal $v$ to the error signal $\varepsilon_{v}, E(s)$, is given by (3), likewise the relationship between $v$ and $q v$ ', $Q(s)$, is detailed in (2).

$$
E(s)=\frac{\varepsilon_{v}}{v}(s)=\frac{s^{2}+\omega^{2}}{s^{2}+k \omega^{\prime} s+\omega^{\prime 2}}
$$

Therefore, a frequency error variable $\varepsilon_{f}$ can be defined as the product of $q v$ ' by $\varepsilon_{v}$. The average value of $\varepsilon_{f}$ will be positive when $\omega<\omega^{\prime}$, zero when $\omega=\omega^{\prime}$ and negative when $\omega \omega^{\prime}$. Hence, as shown in Fig. 2, an integral controller with a negative gain $-\gamma$, can be used to make zero the dc component of the frequency error by shifting the SOGI resonance frequency $\omega^{\prime}$ until matching the input frequency $\omega$. Since the building blocks of the frequency adaptive system of Fig. 2 are the SOGI and the FLL, it was named as the SOGI-FLL.

\section{A. Linearization of the FLL}

As demonstrated in [14], the averaged dynamics of the FLL with $\omega^{\prime} \approx \omega$ can be described by (4).

$$
\dot{\bar{\omega}}^{\prime}=-\frac{\gamma V^{2}}{k \omega^{\prime}}\left(\bar{\omega}^{\prime}-\omega\right)
$$

The equation (4) discloses the relationship between the dynamic response of the FLL and the grid variables and SOGI-QSG gain. From (4), the value of $\gamma$ can be normalized according (5) in order to obtain the linearized system of Fig. 3 , which is nondependent on neither the grid variables nor the SOGI-QSG gain.

$$
\gamma=\frac{k \omega^{\prime}}{V^{2}} \Gamma
$$

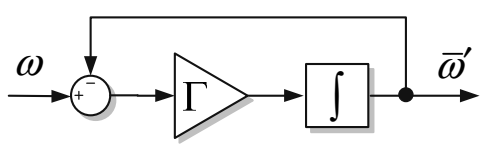

Fig. 3. Simplified frequency adaptation system of the FLL.

The transfer function of the first-order frequency adaptation loop of Fig. 3 is given by:

$$
\frac{\bar{\omega}^{\prime}}{\omega}=\frac{\Gamma}{s+\Gamma} .
$$

Therefore, the settle time is exclusively dependent on the design parameter $\Gamma$ and can be approximated by:

$$
t_{s(F L L)} \approx \frac{5}{\Gamma} .
$$

A practical implementation of the feedback-based linearized FLL is shown in Fig. 4. In this system, the FLL gain is online adjusted by feeding-back the estimated grid operating conditions, which guarantees a constant settle time in grid frequency estimation independently of input signal characteristics.

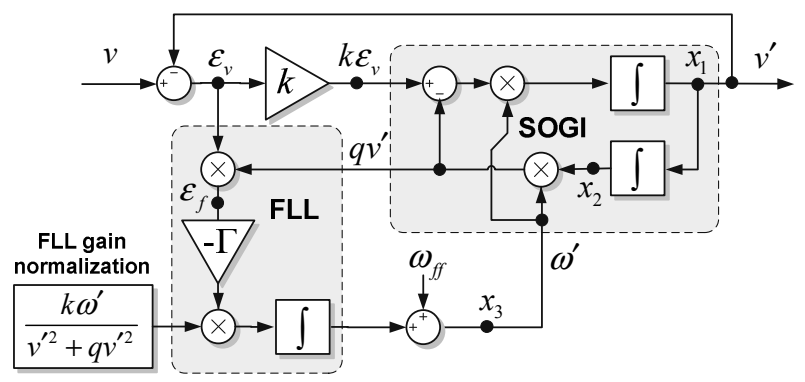

Fig. 4. SOGI-FLL with FLL gain normalization.

\section{DISCRETE IMPLEMENTATION OF THE SOGI-FLL}

Considering that the grid synchronization algorithms are normally programmed in microprocessor, the discrete representation of the SOGI-FLL will be presented as follows.

Among different techniques for discretizing continuous systems, a bilinear transform of the derivative term, such as Tustin approximation, is the best choice when matching the frequency response of the continuous-time system is a matter of interest. This approximation is very suitable for discretizing the SOGI-FLL, a pure resonant system in which the effect of the aliasing in the frequency response should be cancelled in the discretization process, mainly when sampling time increases. Therefore, the SOGI continuous-time statespace equations: 


$$
\begin{gathered}
\dot{\mathbf{x}}=\left[\begin{array}{l}
\dot{x}_{1} \\
\dot{x}_{2}
\end{array}\right]=\mathbf{A} \mathbf{x}+\mathbf{B} v=\left[\begin{array}{cc}
-k \omega^{\prime} & -\omega^{\prime 2} \\
1 & 0
\end{array}\right]\left[\begin{array}{l}
x_{1} \\
x_{2}
\end{array}\right]+\left[\begin{array}{c}
k \omega^{\prime} \\
0
\end{array}\right] v, \\
\mathbf{y}=\left[\begin{array}{c}
v^{\prime} \\
q v^{\prime}
\end{array}\right]=\mathbf{C x}=\left[\begin{array}{cc}
1 & 0 \\
0 & \omega^{\prime}
\end{array}\right]\left[\begin{array}{l}
x_{1} \\
x_{2}
\end{array}\right], \\
\dot{\omega}^{\prime}=-\gamma x_{2} \omega^{\prime}\left(v-x_{1}\right) .
\end{gathered}
$$

will be discretized by using $\dot{x} \approx s^{\prime} x$ approximated functions with:

$$
s^{\prime}=\frac{2}{T_{s}} \frac{z-1}{z+1},
$$

being $T_{s}$ the sampling period. As a result, the SOGI algorithm can be implemented in a digital signal processor by using the following discrete state-space equations:

$$
\begin{gathered}
\mathbf{x}_{[n+1]}=\mathbf{A}^{\prime} \mathbf{x}_{[n]}+\mathbf{B}^{\prime} v_{[n]}, \\
\mathbf{y}_{[n]}=\mathbf{C}^{\prime} \mathbf{x}_{[n]}+\mathbf{D}^{\prime} v_{[n]},
\end{gathered}
$$

being the matrices A', B', C' and D' detailed in (14)-(18). It worth to highlight that the value of $\omega^{\prime}$ in these matrices will be updated with the frequency estimated by the FLL algorithm every computation step.

$$
\begin{aligned}
& \mathbf{A}^{\prime}=\left(\mathbf{I}+\frac{T_{S}}{2} \mathbf{A}\right)\left(\mathbf{I}-\frac{T_{S}}{2} \mathbf{A}\right)^{-1} \\
& \mathbf{A}^{\prime}=\frac{1}{\Delta}\left[\begin{array}{cc}
4-2 T_{S} k \omega_{[n-1]}^{\prime}-T_{S}^{2} \cdot \omega_{n-1]}^{2} & -4 T_{s} \omega_{[n-1]}^{2} \\
4 T_{S} & 4+2 T_{S} k \omega_{n-1]}^{\prime}-T_{S}^{2} \omega_{[n-1]}^{2}
\end{array}\right] \\
& \mathbf{B}^{\prime}=\left(\mathbf{I}-\frac{T_{S}}{2} \mathbf{A}\right)^{-1} \mathbf{B}=\frac{1}{\Delta}\left[\begin{array}{c}
4 k \omega_{[n-1]}^{\prime} \\
2 T_{S} k \omega_{[n-1]}^{\prime}
\end{array}\right], \\
& \mathbf{C}^{\prime}=T_{S} \mathbf{C}\left(\mathbf{I}-\frac{T_{S}}{2} \mathbf{A}\right)^{-1} \\
& \mathbf{C}^{\prime}=\frac{1}{\Delta}\left[\begin{array}{cc}
4 T_{S} & -2 T_{S}^{2} \omega_{[n-1]}^{2} \\
2 T_{S}^{2} \omega_{n-1]}^{\prime} & 2 T_{S} \omega_{[n-1]}^{\prime}\left(2+T_{S} k \omega_{n-1]}^{\prime}\right)
\end{array}\right], \\
& \mathbf{D}^{\prime}=\mathbf{C}\left(\mathbf{I}-\frac{T_{S}}{2} \mathbf{A}\right)^{-1} \frac{T_{S}}{2} \mathbf{B} \\
& \mathbf{D}^{\prime}=\frac{1}{\Delta}\left[\begin{array}{c}
2 T_{S} k \omega_{[n-1]}^{\prime} \\
k T_{S}^{2} \omega_{n-1]}^{\prime 2}
\end{array}\right] \\
& \Delta=4+2 T_{S} k \omega_{[n-1]}^{\prime}+T_{S}^{2} \omega_{[n-1]}^{2}
\end{aligned}
$$

The FLL algorithm is executed after processing the SOGI algorithm and it is discretized by using a trapezoidal integrator, which results in the following discrete equations:

$$
\begin{gathered}
\omega_{[n]}^{\prime}=\omega_{[n-1]}^{\prime}+\frac{T_{s}}{2} \gamma_{[n]}\left(\varepsilon_{f[n]}+\varepsilon_{f[n-1]}\right), \\
\varepsilon_{f[n]}=x_{2[n]}\left(v_{[n]}-v_{[n]}^{\prime}\right), \\
\gamma_{[n]}=\Gamma \frac{k \omega_{[n-1]}^{\prime}}{v_{[n-1]}^{\prime 2}+q v_{[n-1]}^{\prime 2}}
\end{gathered}
$$

The initial values of for the SOGI-FLL algorithm are:

$$
x_{1}=x_{2}=0 ; \omega_{00]}^{\prime}=\omega_{f f},
$$

\section{The DSOGI-FLL}

The SOGI-FLL is a single phase grid synchronization system. However, by means of combining two SOGI-QSG and a single FLL it is possible to obtain a structure for threephase applications.

Considering that most of the three-phase grid-connected power converters employ a three-wire connection, it is only necessary to be synchronized with the positive- and negativesequence components of the grid voltage. This feature permits to transform the grid voltage vector from the $a b c$ to the $\alpha \beta$ stationary reference frames by using the Clarke transformation as indicated in (15).

$$
\mathbf{v}_{\alpha \beta}=\left[T_{\alpha \beta}\right] \mathbf{v}_{a b c} ;\left[T_{\alpha \beta}\right]=\sqrt{\frac{2}{3}}\left[\begin{array}{ccc}
1 & -\frac{1}{2} & -\frac{1}{2} \\
0 & \frac{\sqrt{3}}{2} & -\frac{\sqrt{3}}{2}
\end{array}\right]
$$

In turn, the instantaneous positive- and negative-sequence voltage components on the $\alpha \beta$ reference frame can be calculated as shown in (16), where $q=e^{-j \pi / 2}$ is a 90-degreeslagging phase-shifting operator applied on the time-domain to obtain an in-quadrature version of the input waveforms.

Since the SOGI-QSG behaves as a quadrature signal generator itself, the in-quadrature signals to be computed in (16) in order to calculate the instantaneous positive and negative sequence components of the three-phase input vector can be obtained by means of using a SOGI-QSG for each $\alpha$ and $\beta$ components of the transformed input vector.

$$
\begin{aligned}
\mathbf{v}_{\alpha \beta}^{+} & =\left[T_{\alpha \beta}\right] \mathbf{v}_{a b c}^{+}=\left[T_{\alpha \beta}\right]\left[T_{+}\right] \mathbf{v}_{a b c} \\
& =\left[T_{\alpha \beta}\right]\left[T_{+}\right]\left[T_{\alpha \beta}\right]^{T} \mathbf{v}_{\alpha \beta}=\frac{1}{2}\left[\begin{array}{cc}
1 & -q \\
q & 1
\end{array}\right] \mathbf{v}_{\alpha \beta}, \\
\mathbf{v}_{\alpha \beta}^{-} & =\left[T_{\alpha \beta}\right] \mathbf{v}_{a b c}^{-}=\left[T_{\alpha \beta}\right]\left[T_{-}\right] \mathbf{v}_{a b c} \\
& =\left[T_{\alpha \beta}\right]\left[T_{-}\right]\left[T_{\alpha \beta}\right]^{T} \mathbf{v}_{\alpha \beta}=\frac{1}{2}\left[\begin{array}{cc}
1 & q \\
-q & 1
\end{array}\right] \mathbf{v}_{\alpha \beta},
\end{aligned}
$$


This reasoning permits to obtain the synchronization structure of Fig. 5, which is able to detect the positive- and negative-sequence components of a three-phase input vector at a certain frequency $\omega^{\prime}$.

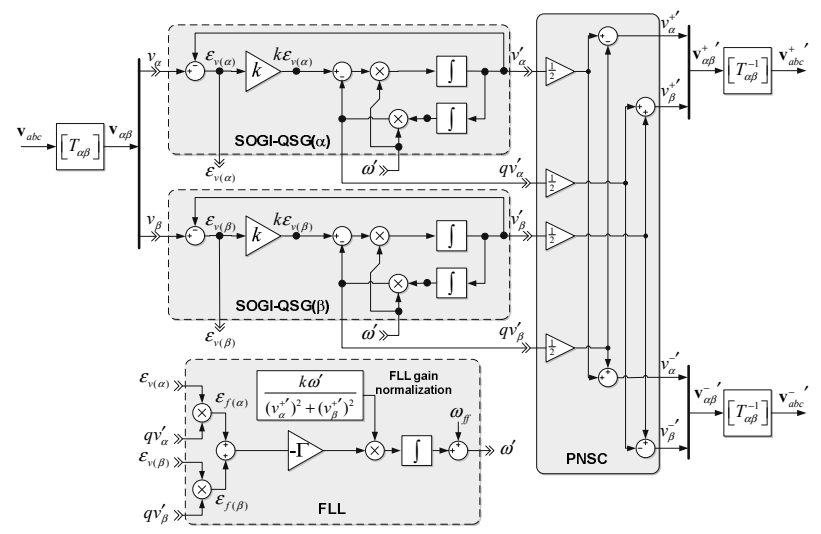

Fig. 5. Block diagram of the DSOGI-FLL divided into its building blocks: SOGI-QSG $(\beta)$, SOGI-QSG $(\alpha)$, FLL and PNSC.

In the system of Fig, 10, two SOGI-QSGs working on the $\alpha \beta$ stationary reference frame provide the inputs signals to a positive-/negative-sequence calculation block (PNSC), which implements the transformations of (16). This system was extensively discussed in [13] and named as Dual SOGI-FLL (DSOGI-FLL).

\section{PERFormance OF THE DSOGI-FLL}

Although the DSOGI-FLL has an inherent pass band filtering behavior, it is not able to estimate accurately the symmetrical components of the fundamental frequency signal if there is an important harmonic distortion in the input. The filtering capability of a single DSOGI-FLL, tuned at $50 \mathrm{~Hz}$, is presented in Fig. 6.

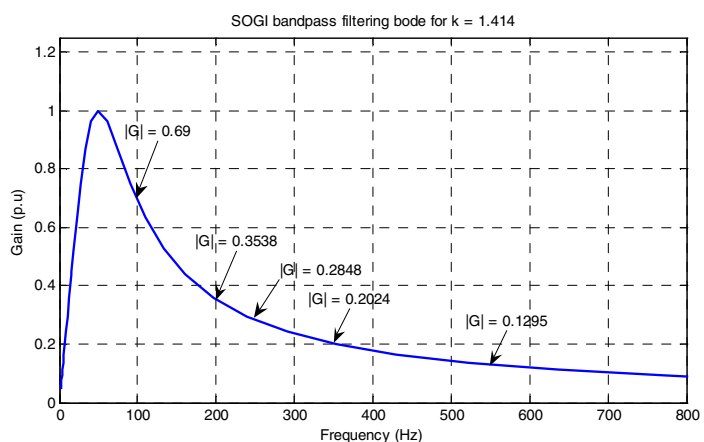

Fig. 6. Filtering capability of the DSOGI-FLL in function of the harmonic order.

This feature has been tested in simulation, considering the DSOGI-FLL structure, shown in Fig. 5, where the input signal has a THD of $40 \%$. This signal has been depicted in Fig, 7.

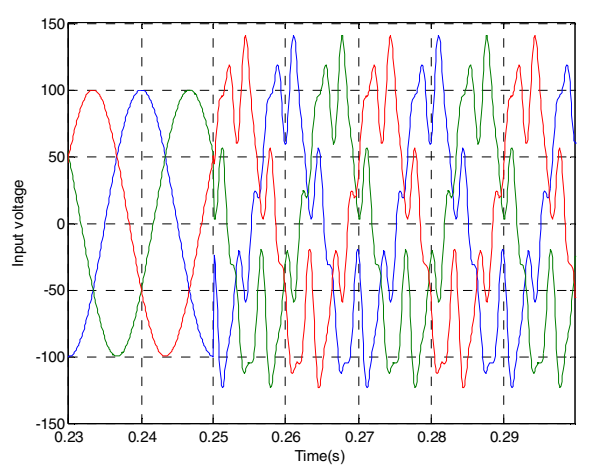

Fig. 7. Testing signal with a THD $=40 \%$.

After processing the signal of Fig. 7 the estimation of the fundamental frequency component, performed by the DSOGI-FLL, gave rise to the waveforms shown in Fig. 8, whose FFT is represented as well.

In can be noticed from the figure how the DSOGI is not
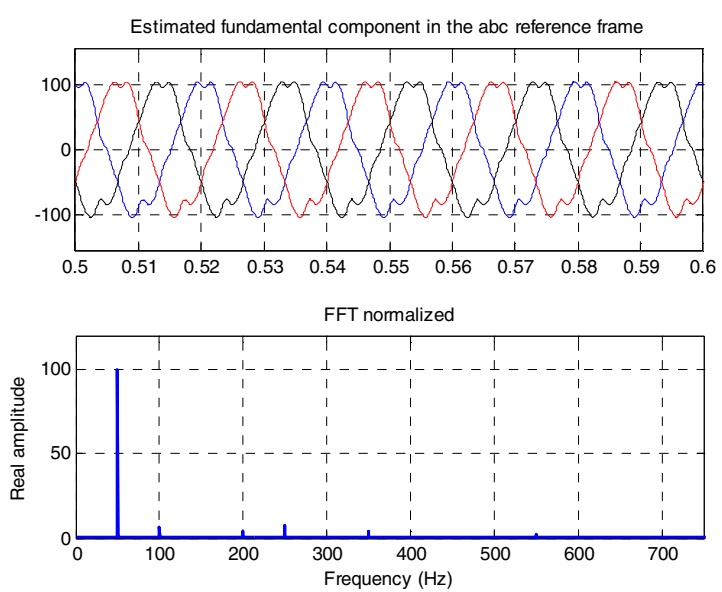

Fig. 8. Estimation of the fundamental component using the DSOGI-FLL

able to extract a sinusoidal signal, due to the high distortion of the input. The reliability of this test can be proven by means of comparing the theoretical and simulated values concerning the harmonic content of the resulting signal, shown in the following TABLE I.

TABLE I

FILTERING CAPABILITY OF THE DSOGI-FLL

\begin{tabular}{|c|c|c|c|}
\hline Order & $\begin{array}{c}\text { Input } \\
\text { harmonic }\end{array}$ & $\begin{array}{c}\text { Simulated } \\
\text { output }\end{array}$ & $\begin{array}{c}\text { Theoretic } \\
\text { output }\end{array}$ \\
\hline $2^{\text {nd }}$ & $10 \%$ & $6.802 \%$ & $6.9 \%$ \\
\hline $3^{\text {rd }}$ & $10 \%$ & $0 \%$ & $0 \%$ \\
\hline $4^{\text {th }}$ & $10 \%$ & $3.474 \%$ & $3.538 \%$ \\
\hline $5^{\text {th }}$ & $25 \%$ & $7.188 \%$ & $7.12 \%$ \\
\hline $7^{\text {th }}$ & $20 \%$ & $4.076 \%$ & $4.048 \%$ \\
\hline $11^{\text {th }}$ & $15 \%$ & $1.967 \%$ & $1.9425 \%$ \\
\hline
\end{tabular}




\section{The Multiple SOGI.FLL}

In this section, a cross-feedback network consisting of multiple ' $n$ ' individual DSOGI-QSGs, like the one shown in Fig. 5, tuned at different frequencies, and working in a collaborative way, is presented as an effective solution to accurately detect the sequence components of the grid voltage, even under very extreme distortion conditions. This new detection system will be referred from now on as the Multiple SOGI-FLL (MSOGI-FLL). The main structure of the MSOGI-FLL is presented in Fig. 9.

As it can be noticed from the figure the MSOGI-FLL can be understood as a set of $n$ selective and adaptive filters, tuned at different frequencies, working in parallel. The FLL block is connected only to the DSOGI-QSG-1, in order to detect the fundamental frequency of the input signal. Then, the estimated frequency is used to tune the frequency of each of the DSOGI-QSGs, by multiplying its value by a coefficient which determines the order of the harmonic to be detected by each DSOGI-QSG. This structure permits thus to detect the different harmonic components of the input signal.

The accuracy of this estimation can be highly affected when the harmonic components are in a narrow range, due to the intrinsic bandwidth of the DSOGI-QSG. In order to overcome this drawback the MSOGI-FLL presents an additional and extremely relevant advantage resulting from the cross-feedback network.

As shown in Fig. 9, the input signal of each DSOGI-QSG results from subtracting the output of all the rest of DSOGIQSGs from the original input signal $v$. In this way, after a transient process, the input signal of each DSOGI-QSG will be cleaned up from the harmonic components detected by the rest of DSOGI-QSGs, which will contribute to attenuate the distortion at their outputs.

The Bode diagram for the DSOGI-QSG- $i$, shown in Fig. 10 , for a MSOGI-FLL, consisting of four individual DSOGIQSGs tuned at the $2^{\text {nd }}, 4^{\text {th }}, 5^{\text {th }}$ and $7^{\text {th }}$ harmonics, show how the cross-feedback network of the MSOGI-FLL gives rise to notches in the frequency response curve at the frequencies which the individual DSOGI-QSG are tuned to.

As a consequence, the selective filtering characteristic of

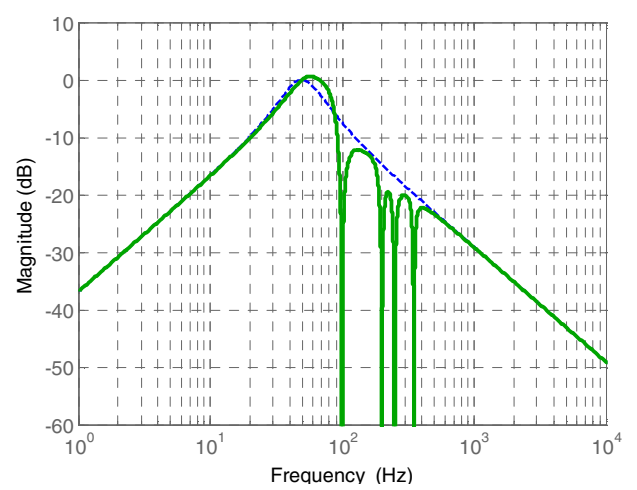

Fig. 10. Frequency response of the single-phase MSOGIFLL.

each DSOGI-QSG is improved and its response is enhanced in case of high distortion level on the input voltage if compared with the dashed line in Fig. 10, which represents the frequency response curve for the case in which the crossfeedback network is disabled.

This statement can be proven easily by means of applying

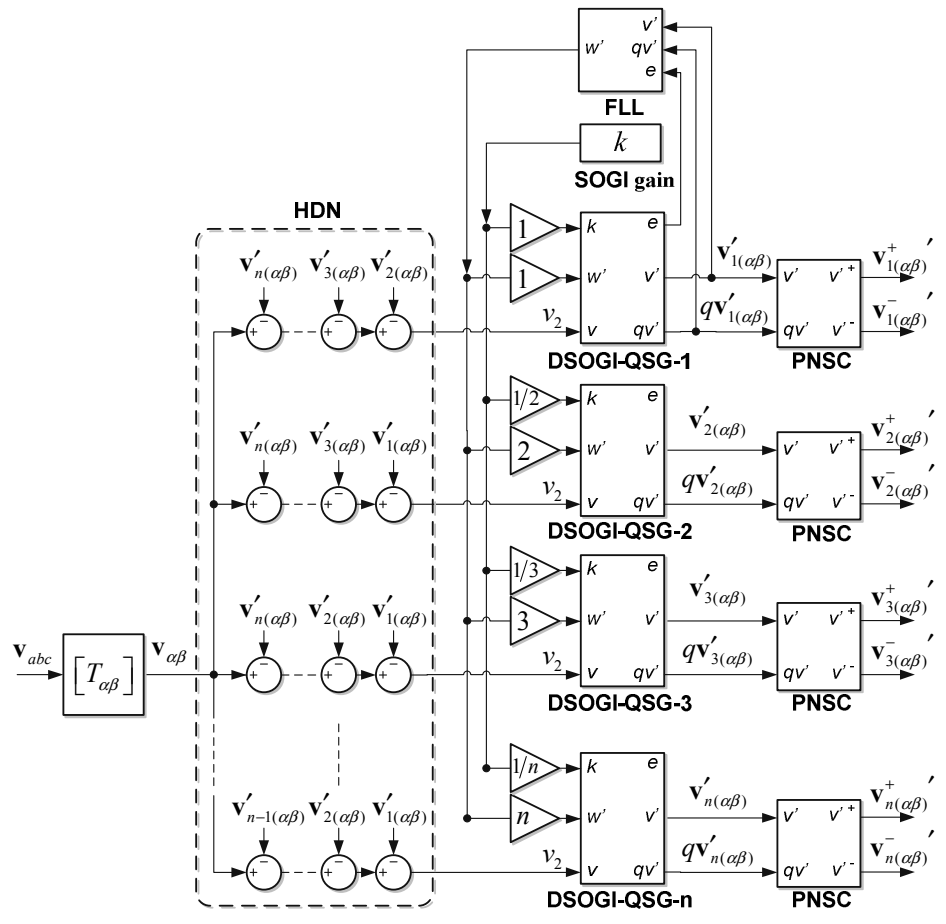

Fig. 9. MSOGI-FLL for three-phase systems able to synchronize with ' $n$ ' harmonic components of the input vector. 
the testing signal of Fig. 7 to the input of the MSOGI. The results obtained in this case are shown in the following figure.

The waveforms in Fig.11 stand out clearly the better
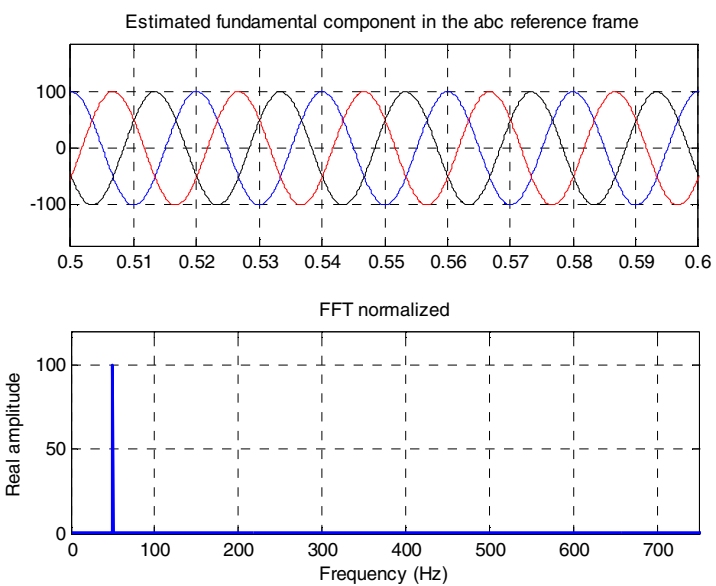

Fig. 11. The SOGI-FLL, a single-phase grid synchronization system.

behavior of the MSOGI if compared with a single DSOGI response, displayed in Fig.8.

\section{EXPERIMENTAL RESULTS}

To experimentally validate the performance of the synchronization system presented in this paper, the algorithm of the MSOGI-FLL was implemented in a control board based on the floating point DSP Texas Instruments TMS320F28335 at $150 \mathrm{MHz}$. The unbalanced and distorted input voltage was generated by means of an $\mathrm{AC}$ programmable source ELGAR SM5250A.

The MSOGI-FLL was implemented with seven embedded DSOGIs (one per each harmonic plus the fundamental one), as detailed in TABLE II.

In this study case, the sampling frequency was set to 10

TABLE II

PARAMETERS OF THE INPUT VOLTAGE

\begin{tabular}{cc}
\hline Voltage component & Value [p.u.] \\
\hline Fundamental positive-sequence & $\vec{V}^{+1}=0.733 \angle 5^{\circ}$ \\
Fundamental negative-sequence & $\vec{V}^{+1}=0.210 \angle 50.4^{\circ}$ \\
$2^{\text {nd }}$ harmonic negative-sequence & $\vec{V}^{-2}=0.10 \angle 0^{\circ}$ \\
$3^{\text {rd }}$ harmonic zero-sequence & $\vec{V}^{03}=0.10 \angle 45^{\circ}$ \\
$4^{\text {th }}$ harmonic positive-sequence & $\vec{V}^{+4}=0.10 \angle 180^{\circ}$ \\
$5^{\text {th }}$ harmonic negative-sequence & $\vec{V}^{-5}=0.25 \angle 45^{\circ}$ \\
$7^{\text {th }}$ harmonic positive-sequence & $\vec{V}^{+7}=0.20 \angle 180^{\circ}$ \\
$11^{\text {th }}$ harmonic negative-sequence & $\vec{V}^{-11}=0.15 \angle 180^{\circ}$ \\
\hline
\end{tabular}

$\mathrm{kHz}$ and the computational cost of the whole MSOGI-FLL took $34.86 \mu \mathrm{s}$.

Fig. 12 shows some scopes recorded from the control board by using the PWM outputs and a low-pass filters as digital-toanalog converters [15].

Fig. 12(a) shows the input voltage, which was unbalanced and extremely distorted. Figs. 12(b) and 12(c) show the waveforms detected for the positive- and negative-sequence components at the fundamental frequency. Figs. 12(d), 12(e), 12(f), 12(g) and 12(h) show the waveforms detected for the $2 \mathrm{nd}, 4 \mathrm{th}, 5$ th, 7 th and 11 th harmonics, respectively.
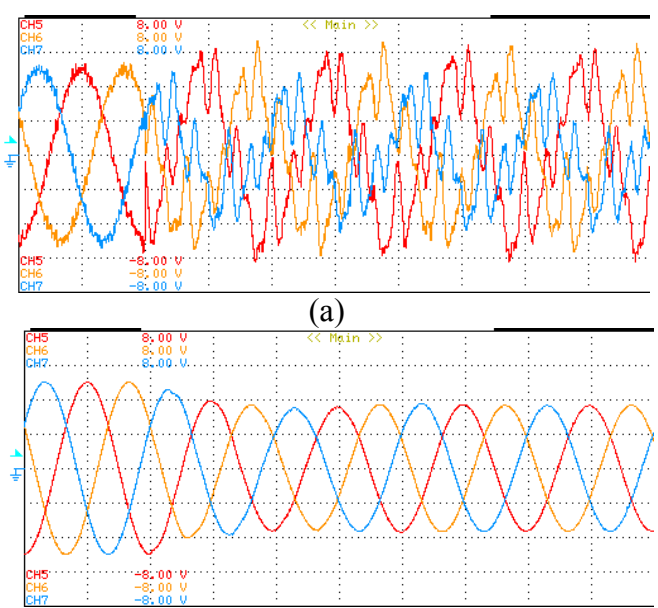

(b)
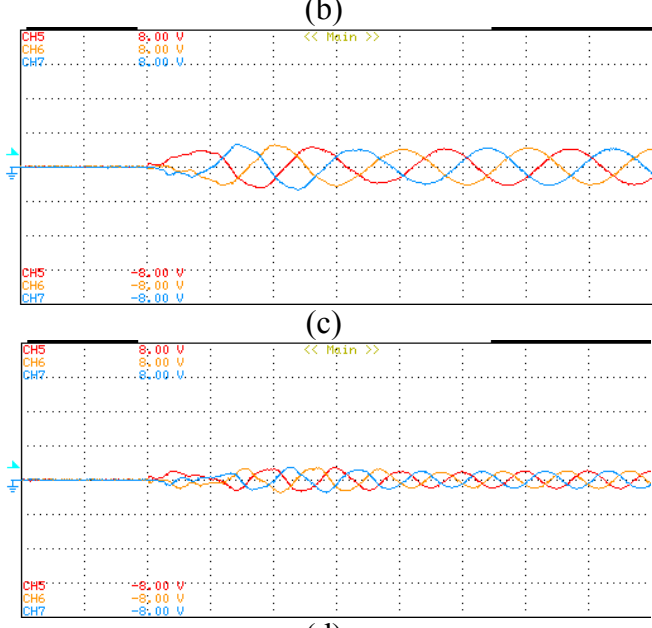

(d)

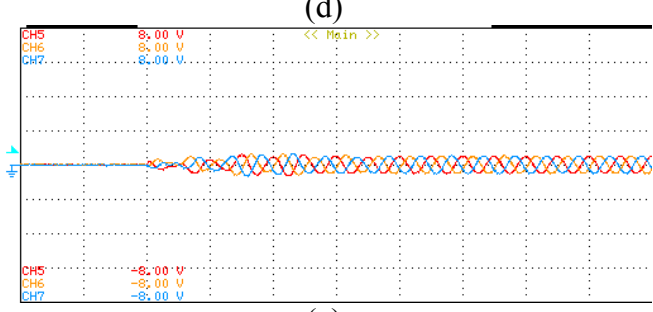

(e) 


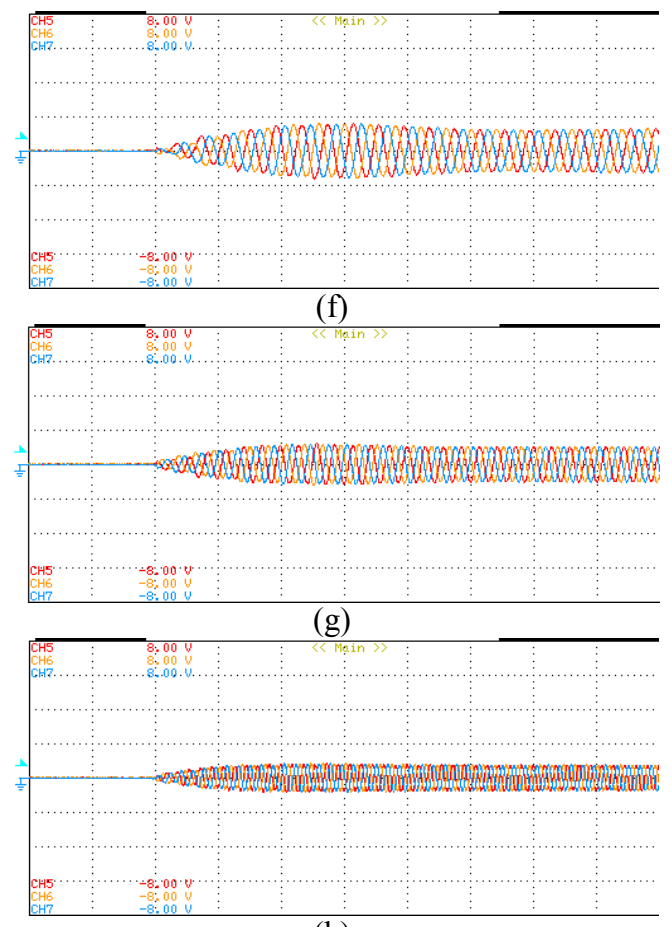

(h)

Fig. 12.- Experimental response of the MSOGI-FLL.

According to the results shown in Fig. 12, it should be pointed out the excellent performance of the MSOGI-FLL.

\section{CONCLUSIONS}

The MSOGI-FLL permits detecting the positive and negative component of each harmonic of the measured grid voltage under extremely polluted conditions.

As shown in the paper, the resulting MSOGI-FLL is a frequency-adaptive algorithm thanks to the FLL block, that permits detecting directly the fundamental frequency of the voltage/current. This feature is an additional advantage of this synchronization system, if compared with other advanced PLLs, as the grid frequency variable is more stable than voltage phase-angle during transient events.

Regarding its structure it should be pointed out that the MSOGI-FLL does not need to perform any trigonometric function, since neither synchronous reference frame nor voltage controlled oscillator are used in its algorithm. This feature contributes to reduce its computational cost, getting finally a low computational burden time algorithm.

The simulated and experimental results presented in this work shows that the MSOGI-FLL is a very suitable solution to the detection of fundamental-frequency positive- and negative-sequence components of unbalanced and distorted grid voltages.

\section{ACKNOWLEDGMENT}

This work was supported by the projects ENE2008-06841C02-01/ALT and ENE2008-06588-C04-03/ALT financed by the Ministerio de Ciencia e Innovacion of Spain.

\section{REFERENCES}

[1] "Towards Smart Power Networks - Lessons learned from European research FP5 projects", European Commission - Office for Official Publications of the European Communities, Ref. EUR 21970, Luxembourg, 2005

[2] S. Chung, "A phase tracking system for three phase utility interface inverters," IEEE Trans. Power Electron., vol. 15, pp. 431-438, May 2000.

[3] P. Rodriguez, J. Pou, J. Bergas, J.I. Candela, R.P. Burgos, and D. Boroyevich, "Decoupled Double Synchronous Reference Frame PLL for Power Converters Control," IEEE Trans. Power Electron., vol.22, no.2, pp.584-592, Mar. 2007.

[4] P. Rodríguez, A. Luna, R. Teodorescu, F. Blaabjerg., "Grid Synchronization of Wind Turbine Converters under Transient Grid Faults using a Double Synchronous Reference Frame PLL". Energy 2030 Conference, 2008.

[5] F. Blaabjerg, R. Teodorescu, M. Liserre, A.V. Timbus, "Overview of Control and Grid Synchronization for Distributed Power Generation Systems," IEEE Trans. on Industrial Electronics, vol. 53, no. 5, pp. 1398-1409, Oct 2006

[6] V.M. Moreno, M. Liserre, A. Pigazo, A. Dell'Aquila, "A Comparative Analysis of Real-Time Algorithms for Power Signal Decomposition in Multiple Synchronous Reference Frames," IEEE Trans. Power Electron., vol.22, no.4, pp.1280-1289, July 2007.

[7] M. Karimi-Ghartemani and M.R. Iravani, "A method for synchronization of power electronic converters in polluted and variable-frequency environments," IEEE Trans. Power Systems, vol. 19, pp. 1263-1270, Aug. 2004.

[8] D. Yazdani, A. Bakhshai, G. Joos, M. Mojiri, "A Nonlinear Adaptive Synchronization Technique for Grid-Connected Distributed Energy Sources," IEEE Trans. Power Electron., vol.23, no.4, pp.2181-2186, July 2008.

[9] M. Mojiri, M. Karimi-Ghartemani, A. Bakhshai, "Time-Domain Signal Analysis Using Adaptive Notch Filter," IEEE Transactions on Signal Processing, vol.55, no.1, pp.85-93, Jan. 2007

[10] K. de Brabandere, T. Loix, K. Engelen, B. Bolsens, J. Van den Keybus, J. Driesen, and R. Belmans, "Design and Operation of a Phase-Locked Loop with Kalman Estimator-Based Filter for Single-Phase Applications," in Proc. IEEE Ind. Electron. Conf. (IECON'06), Nov. 2006, pp. 525-530.

[11] P. Rodriguez, R. Teodorescu, I. Candela, A.V. Timbus, M. Liserre, and F. Blaabjerg, "New Positive-sequence Voltage Detector for Grid Synchronization of Power Converters under Faulty Grid Conditions," in Proc. IEEE Power Electron. Spec. Conf. (PESC'06), Jun. 2006, pp. 1-7.

[12] M. Ciobotaru, R. Teodorescu, and F. Blaabjerg, " A New Single-Phase PLL Structure Based on Second Order Generalized Integrator," in Proc. IEEE Power Electron. Spec. Conf. (PESC'06), Jun. 2006, pp. 1-7.

[13] P. Rodriguez, A. Luna, M. Ciobotaru, R. Teodorescu, and F. Blaabjerg, "Advanced Grid Synchronization System for Power Converters under Unbalanced and Distorted Operating Conditions," in Proc. IEEE Ind. Electron. Conf. (IECON'06), Nov. 2006, pp.5173-5178.

[14] P. Rodríguez, A. Luna,I. Candela, R. Teodorescu, F. Blaabjerg., "Grid synchronization of power converters using multiple second order generalized integrators". Industrial Electronics, 2008. IECON 2008. 34th Annual Conference of IEEE

[15] D. M. Alter, "Using PWM output as a Digital-to-nalog converter on a TMS320F280X digital signal controller," Application report SPAA88, Texas Instruments, Feb. 2006, pp. 1-30. 
2246 